\title{
Are Exercise Referral Schemes Associated With an Increase in Physical Activity? Observational Findings Using Individual Patient Data Meta-Analysis From the National Referral Database
}

\author{
Nikita Rowley, James Steele, Matthew Wade, Robert James Copeland, Steve Mann, \\ Gary Liguori, Elizabeth Horton, and Alfonso Jimenez
}

\begin{abstract}
Objectives: To examine if exercise referral schemes (ERSs) are associated with meaningful changes in physical activity in a large cohort of individuals throughout England, Scotland, and Wales from The National Referral Database. Methods: Data were obtained from 5246 participants from 12 different ERSs, lasting 6-12 weeks. The preexercise referral scheme and changes from the preexercise to the postexercise referral scheme in self-reported International Physical Activity Questionnaire scores were examined. A 2-stage individual patient data meta-analysis was used to generate the effect estimates. Results: For the pre-ERS metabolic equivalent (MET) minutes per week, the estimate (95\% confidence interval [CI]) was 676 MET minutes per week (539 to 812). For the change in MET minutes per week, the estimate (95\% CI) was an increase of 540 MET minutes per week (396 to 684). Changes in the total PA levels occurred as a result of increases in vigorous activity of 17 minutes (95\% CI, 9 to 24), increases in moderate activity of 29 minutes (95\% CI, 22 to 36), and reductions in sitting of -61 minutes (95\% CI, -78 to -43 ), though little change in walking ( $-5 \mathrm{~min} ; 95 \% \mathrm{CI}$, -14 to 5) was found. Conclusions: Most participants undergoing ERSs are already "moderately active." Changes in PA behavior associated with participation are through increased moderate to vigorous PA and reduced sitting. However, this was insufficient to change the International Physical Activity Questionnaire category, and the participants were still "moderately active."
\end{abstract}

Keywords: health database, IPAQ, health behavior

\section{What is Already Known on This Topic?}

- Physical activity (PA) is widely considered to be effective in the prevention, management, and treatment of many chronic health disorders, yet population PA levels are relatively low and have changed little in recent years.

- Sufficient PA levels for health and well-being often do not arise as a result of typical activities of daily living, and thus, specific exercise has been argued to be necessary for many, and one approach to providing this has been through exercise referral schemes (ERSs).

- ERSs are aimed at increasing PA levels in sedentary individuals with chronic disease; however, despite evidence of the benefits of PA, the evidence base regarding whether these are an effective approach to increase PA is currently limited.

\section{What This Study Adds}

- Our findings suggest that ERSs may not be targeting the population for which they are aimed, as participants are

Rowley, Horton, and Jimenez are with the Centre for Innovative Research Across the Life Course (CIRAL), Faculty of Health and Life Sciences, Coventry University, Coventry, United Kingdom. Steele, Wade, Mann, and Jimenez are with the ukactive Research Institute, London, United Kingdom. Steele is also with the School of Sport, Health, and Social Sciences, Solent University, Southampton, United Kingdom. Wade is also with St Mary's University, London, United Kingdom. Copeland is with the National Centre for Sport and Exercise Medicine, Sheffield, United Kingdom; and Sheffield Hallam University, Sheffield, United Kingdom. Mann is also with Places for People Leisure, London, United Kingdom. Liguori is with The University of Rhode Island, Kingston, RI, USA. Jimenez is also with the GO fit LAB, Ingesport, Madrid, Spain. Rowley (pricen5@coventry.ac.uk) is corresponding author. typically classified as "moderately active" prior to beginning their exercise referral scheme, or that participants are overestimating their levels of PA.

- ERSs are associated with a statistically significant change in total PA, with most of this accounted for by increases in moderate-vigorous PA (increasing $17 \mathrm{~min}$ and $29 \mathrm{~min} / \mathrm{wk}$, respectively), in addition to reductions in sitting time (reducing by $61 \mathrm{~min} / \mathrm{wk}$ ); however, the size of the changes was not sufficient for the participants to move from the "moderately active" category to the "highly active" category.

- These findings suggest the need to consider ERSs and their implementation more critically. It would seem that they may not be targeting those who are most inactive or that participants are overestimating their PA levels at the baseline, and this may explain why the changes were not sufficient to change the activity category.

Physical activity is widely considered to be an effective prevention and management tool for a wide range of chronic health disorders. ${ }^{1-3}$ PA is considered to be any bodily movement created by skeletal muscles that results in a greater demand of energy expenditure than would normally be required. ${ }^{4}$ PA can be conducted in many ways, including unstructured activities as part of an individual's daily living, leisure activities, or occupation, and it is often performed without the explicitly desired goal of improving fitness. Improving health and fitness can be a by-product of these unstructured activities, although unstructured PA is decreasing within the modern era. ${ }^{5}$

Worldwide, 1 in 4 adults do not meet the current global recommendations for PA, which suggest that adults should undertake 150 minutes of moderate-intensity activity per week. ${ }^{4}$ Approximately 20 million adults in the United Kingdom are not physically active, ${ }^{6}$ a figure that has remained relatively unchanged in recent 
years. ${ }^{7}$ Physical inactivity is a public health dilemma in that it is associated with an increased risk of noncommunicable diseases, including obesity, cardiovascular diseases, diabetes, and premature death. ${ }^{7}$ Physical inactivity has reportedly increased globally, having serious consequences on health and well-being. ${ }^{7-9}$ In contrast to inactivity, which is associated with a range of negative health outcomes, PA is associated with a range of positive health outcomes. $^{2,5}$ Indeed, network meta-analyses have shown that PA interventions, including structured bouts of PA (ie, exercise), are similarly effective as, and in some cases more effective than, drug treatments for secondary prevention. ${ }^{10,11}$

Considering this, interventions to increase PA in primary care might present a solution to reduce the heavy burden that inactivityrelated noncommunicable diseases place upon the National Health Service, ${ }^{12}$ which at present, has risen to $£ 1.2$ billion per year. ${ }^{7}$ ERSs are exercise interventions aimed at increasing the number of sedentary individuals becoming active, along with aiding the rehabilitation and management of chronic health disorders. ${ }^{1,13,14}$ Schemes were first introduced in the 1990s in primary care settings across England to facilitate PA participation for individuals referred with chronic health disorders. ${ }^{15}$ Professionals in primary care (usually physicians/ general practitioners, but also nurses, physiotherapists, and condition-specific specialists) typically refer individuals to third-party service providers, usually in leisure centers and gyms, who then prescribe an exercise program and monitor progress accordingly.

Guidance for ERSs in England, published by The National Institute for Health and Care Excellence (NICE), "PH54: PA: ERSs,"13 are vague in the details regarding what exercise providers should base their schemes upon. However, they do suggest that schemes typically consist of a 12-week exercise prescription and target inactive individuals with chronic health disorders. Yet, the evidence available for inactive but presently healthy individuals, specifically, was considered weak at the time of the 2014 guidelines, which in recent consultation, have remained the same. ${ }^{16}$ The NICE guidelines do not provide any details of the specific exercise prescriptions used within schemes. In addition, ERSs have been described as "wild and woolly," with a lack of agreement between stakeholders on how to determine impact. ${ }^{17}$ Although increasing PA levels, both during the intervention and resultant from it, is a primary aim of ERSs, there has been little research documenting the change in PA levels after scheme completion; and what has been conducted appears conflicting. ${ }^{1,18-20}$ This is of particular relevance, as recent observational findings reported from ERSs in The National Referral Database suggest that changes in health and well-being outcomes may not reach meaningful levels. ${ }^{21}$ It is important to provide an update of evidence of whether ERSs do impact PA across England, Scotland, and Wales to meet the recommendations from NICE and to understand whether a possible explanation for the lack of health and well-being outcomes may be due to insufficient changes in PA levels. Further research to document changes in PA levels was also a recommendation from NICE. ${ }^{13}$ The aim of this study was therefore to examine whether ERSs are effective in providing change in PA in participants who complete an ERS, using observational data from The National Referral Database to meet the recommendations for further research from NICE and to review data from the first national database in the United Kingdom.

\section{Methods}

\section{Study Design}

Anonymized data were extracted from The National Referral Database after being uploaded from ERSs across England, Wales, and Scotland. Referrals from primary care to ERSs were made between September 2011 and October 2017. At the time of the data cut in October 2017, the data were exported from The National Referral Database into an Excel spreadsheet, where it went through a process of data cleaning to ready it for analysis. The database has been described elsewhere and includes database formation, data cleaning, and structure in detail. ${ }^{22}$ The study used a retrospective cohort longitudinal study design (STROBE checklist available in Supplementary Material [available online]), following individuals entering and exiting ERS after referral from a range of organizations and referrer types (general practitioners, nurses, physiotherapists, and condition-specific specialists) across the United Kingdom. Due to the inclusion of various schemes within the database, in order to account for scheme level variance, a multilevel modeling approach was employed involving an individual patient data random effects meta-analysis with a 2-stage approach (see "Statistical Analysis" section).

\section{Outcome Measures}

The National Referral Database used the self-reported International Physical Activity Questionnaire (IPAQ) short form to measure the PA levels of the participants. This was the PA questionnaire that was used by exercise providers who uploaded data onto the National Referral database throughout the United Kingdom; therefore, this was used to measure PA within this study. This questionnaire was used to determine weekly PA in metabolic equivalent (MET) minutes per week (described below), which was the primary outcome measure. The change in MET minutes per week of self-reported PA prescheme and postscheme was used to examine the impact ERSs had on the participants' PA levels. The IPAQ short form contains 7 open-ended items surrounding the participants' last 7-day recall of PA and sitting behaviors. The items were structured to provide scoring on walking and moderate-intensity and vigorous-intensity activity, in addition to sitting. The IPAQ has been designed for observational research, and its test-retest reliability indicates good stability and high reliability $(\alpha$ $>.80$ ), along with concurrent validity. ${ }^{23,24}$ Both continuous and categorical indicators of PA come from IPAQ.

\section{Continuous Analysis of IPAQ}

Due to the nonnormal distribution of energy expenditure in participants, it has been suggested that continuous indicators be presented as median MET minutes per week. ${ }^{25}$ A MET is the ratio of the rate of energy expended during an activity to the rate of energy expended at rest. ${ }^{26} \mathrm{~A}$ MET is a unit of energy expenditure and, by calculating MET minutes, can be used to track the amount of PA an individual is doing per week. ${ }^{25}$

\section{Categorical Analysis of IPAQ}

There are 3 categorical levels of PA scoring to classify populations through the IPAQ: "low," "moderate," and "high." The criteria set for each of the levels consider each question asked on the IPAQ form. ${ }^{23}$ The "high" category describes high levels of PA participation, either $>1500$ MET minutes per week (consisting of vigorous activity on at least $3 \mathrm{~d}$ ) or $>3000$ MET minutes per week (consisting of any combination of activities across $7 \mathrm{~d}$ ). This provides a higher threshold of measures of total PA and is useful to examine population variation. The "moderate" category (600-1499 MET $\mathrm{min} / \mathrm{wk}$ ) defines an individual participating in some activity more than those in the "low" category. Those in the "low" category do not engage in at least half an hour moderate-intensity PA most days 
(0-599 MET min/wk). Individuals in the "low" category do not meet any criteria from the high or moderate categories and are not participating in any regular PA.

\section{Statistical Analyses}

The analyses were performed with the intention of reporting broadly "do we observe a meaningful change in the PA levels in individuals who are undergoing ERSs?"

A 2-stage individual patient data meta-analysis was performed on both the median pre-ERS and the median change scores (ie, post minus pre-ERS scores) for the MET minutes. An analysis was also performed on the breakdown of vigorous- and moderate-intensity activity, walking, and sitting minutes for pre-ERS, as well as the change scores. For stage 1, both median pre-ERS for MET minutes per week and mean pre-ERS for activity breakdowns, and median change scores for MET minutes per week and mean for activity breakdowns, and their standard errors were derived for each scheme. The second stage involved performing a random effects meta-analysis using the "metafor" package in R (version 3.5.0; $\mathrm{R}$ Core Development Team, https://www.r-project.org/) across all schemes to derive a final point estimate and precision of estimate (95\% confidence intervals [CIs]). A random effects model was used, as our aim was to estimate the PA levels and changes in PA levels for individuals undergoing any ERS. We assumed that, due to there being considerable uncertainty in the manner in which each individual ERS scheme was delivered, it was not a reasonable assumption to treat them all as providing estimates of a fixed or common effect. That is to say, the variation in effect sizes estimated for each scheme was assumed not due to solely sampling variation. Estimates were weighted by inverse sampling variance, and restricted maximal likelihood estimation was used in all models. Schemes without sufficient participants $(n<4)$ were excluded from analysis. Robustness of the main effects was considered through the sensitivity analyses by removal of individual schemes and reanalysis of the random effects model. Where significant estimates became nonsignificant and vice versa, in addition to where there were considerable changes in the magnitude and/or precision of those estimates, the results of the sensitivity analyses are reported.
An $\alpha$ level of .05 was used to determine statistical significance; however, the results were not interpreted dichotomously based purely on this or whether the $95 \%$ CI crossed zero. Instead, the point estimate and its precision were considered in light of the PA guidelines and interpreted with respect to how meaningful the change was. In this sense, progressively greater increases in MET minutes per week are required as starting PA levels increase to move into a higher category. This was based upon the IPAQ "low," "moderate," and "high" categories. For high, we considered the lower threshold of 1500 MET minutes per week. Categorical IPAQ data were also examined, and the descriptive proportions, both preERS and post-ERS, were considered across schemes, in addition to longitudinal plotting across the entire sample to examine changes between the IPAQ categories.

\section{Results}

A total of 12 schemes were included in the final analysis, which included a total of 5246 participants' data, with an average age of 53 (15) years and $68 \%$ of whom were female.

\section{Categorical IPAQ Classification}

An examination of the categorical data revealed that roughly half of the participants who began an ERS were not classified as having "low" levels of PA. There were shifts from pre-ERS to post-ERS in the proportions of participants in each category, with decreases in the proportion of those in the "low" categories and increases in both "moderate" and "high." Table 1 shows the proportions across schemes. Tracking of the entire sample visually (Figure 1) showed that the participants within the "low" categories tended to shift into the "moderate" and "high" categories. Some participants within the "moderate" category also shifted into the "high" category. However, a number of participants in the "low" category remained in this category, some in the "moderate" category dropped into the "low" category, and some in the "high" category dropped into both the "moderate" and "low" categories.

Table 1 Proportions of Participants in IPAQ Categories Pre-ERS and Post-ERS

\begin{tabular}{|c|c|c|c|c|c|c|}
\hline \multirow[b]{2}{*}{ Scheme } & \multicolumn{2}{|c|}{ Low } & \multicolumn{2}{|c|}{ Moderate } & \multicolumn{2}{|c|}{ High } \\
\hline & Pre & Post & Pre & Post & Pre & Post \\
\hline 5001 & $197(36.1 \%)$ & $90(16.5 \%)$ & $286(52.5 \%)$ & $282(51.7 \%)$ & $62(11.4 \%)$ & $173(31.7 \%)$ \\
\hline 5002 & $146(32.4 \%)$ & $47(10.4 \%)$ & $221(49.1 \%)$ & $295(65.6 \%)$ & $61(13.6 \%)$ & $108(24.0 \%)$ \\
\hline 5036 & $615(45.0 \%)$ & $296(24.3 \%)$ & $547(50.6 \%)$ & $752(61.8 \%)$ & $54(4.4 \%)$ & $168(13.8 \%)$ \\
\hline 5056 & $748(58.9 \%)$ & $443(34.9 \%)$ & $459(36.1 \%)$ & $735(57.8 \%)$ & $64(5.0 \%)$ & $93(7.3 \%)$ \\
\hline 5063 & $61(33.0 \%)$ & $26(14.1 \%)$ & $85(45.9 \%)$ & $69(37.3 \%)$ & $32(17.3 \%)$ & $38(20.5 \%)$ \\
\hline 5072 & $325(55.4 \%)$ & $111(18.9 \%)$ & $226(38.5 \%)$ & $371(63.2 \%)$ & $36(6.1 \%)$ & $105(17.9 \%)$ \\
\hline 5089 & $148(71.2 \%)$ & $103(49.5 \%)$ & $60(28.8 \%)$ & $100(48.1 \%)$ & $0(0.0 \%)$ & $5(2.4 \%)$ \\
\hline 5108 & $131(54.8 \%)$ & $51(21.3 \%)$ & $95(39.7 \%)$ & $156(65.3 \%)$ & $13(5.4 \%)$ & $32(13.4 \%)$ \\
\hline 5119 & $3(42.9 \%)$ & $0(0.0 \%)$ & $3(42.9 \%)$ & $3(42.9 \%)$ & $1(14.3 \%)$ & $4(57.1 \%)$ \\
\hline 5131 & $159(38.0 \%)$ & $32(7.7 \%)$ & $199(47.6 \%)$ & $214(51.2 \%)$ & $60(14.4 \%)$ & $172(41.1 \%)$ \\
\hline 5144 & $7(21.2 \%)$ & $0(0.0 \%)$ & $12(36.4 \%)$ & $10(30.3 \%)$ & $13(39.4 \%)$ & $23(69.7 \%)$ \\
\hline 5156 & $55(64.0 \%)$ & $18(20.9 \%)$ & $29(33.7 \%)$ & $52(60.5 \%)$ & $2(2.3 \%)$ & $16(18.6 \%)$ \\
\hline All schemes & $2595(49.5 \%)$ & $1217(23.2 \%)$ & $2223(42.4 \%)$ & $3039(57.9 \%)$ & $398(7.6 \%)$ & $938(17.9 \%)$ \\
\hline
\end{tabular}

Abbreviations: ERS, exercise referral scheme; IPAQ, International Physical Activity Questionnaire. Note: Where percentages do not sum to $100 \%$, this is due to missing data and inability to categorize some participants (ie, they were classified based upon their IPAQ responses as "unknown"). 


\section{Pre-ERS MET Minutes}

For pre-ERS MET minutes per week, the estimate from the random effects model was 676 MET minutes per week (539-812 min, $P<.0001)$. Figure 2 shows the forest plot for pre-ERS MET minutes. Significant heterogeneity was evident among the schemes $\left(Q_{(11)}=84.31, P<.0001 ; I^{2}=90.41 \%\right)$, however, the sensitivity analysis did not reveal any influential schemes.

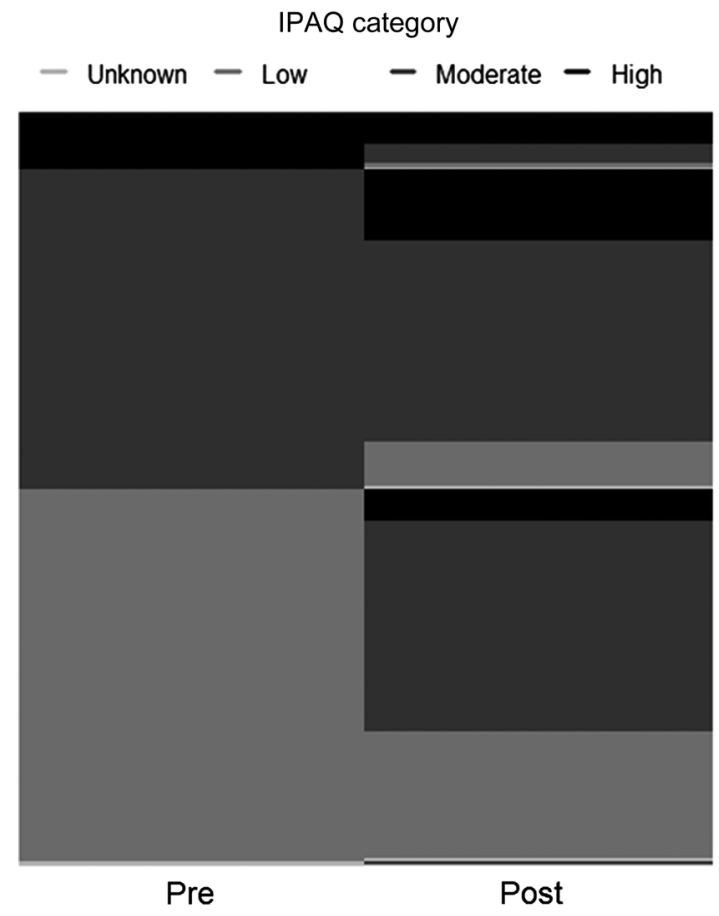

Figure 1 - Pre-ERS and post-ERS changes in categorical IPAQ classification across participants. IPAQ indicates international physical activity questionnaire.

\section{Pre-ERS Breakdown of Activity Minutes}

The forest plots are shown for the pre-ERS breakdown of activity minutes in Figure 3. For pre-ERS vigorous activity, the estimate from the random effects model was 25 minutes (16-34 min, $P<$ $.0001)$. Significant heterogeneity was evident among the schemes $\left(Q_{(10)}=128.54, P<.0001 ; I^{2}=87.52 \%\right)$; however, the sensitivity analysis did not reveal any influential schemes. For pre-ERS moderate activity, the estimate from the random effects model was 45 minutes $(38-51 \mathrm{~min}, P<.0001)$. Significant heterogeneity was evident among the schemes $\left(Q_{(11)}=84.15, P<.0001 ; I^{2}=\right.$ $87.52 \%)$; however, the sensitivity analysis did not reveal any influential schemes. For pre-ERS walking, the estimate from the random effects model was 59 minutes $(48-69 \mathrm{~min}, P<.0001)$. Significant heterogeneity was evident among the schemes $\left(Q_{(11)}=\right.$ $\left.167.73, P<.0001 ; I^{2}=96.66 \%\right)$; however, the sensitivity analysis did not reveal any influential schemes. For pre-ERS sitting, the estimate from the random effects model was 384 minutes (352-415 $\mathrm{min}, P<.0001)$. Significant heterogeneity was evident among the schemes $\left(Q_{(11)}=365.00, P<.0001 ; I^{2}=97.20 \%\right)$; however, the sensitivity analysis did not reveal any influential schemes.

\section{Change in MET Minutes}

For the change in MET minutes per week, the estimate from the random effects model was 540 MET minutes per week (396$684 \mathrm{~min}, P<.0001)$. Figure 4 shows the forest plot for the change in MET minutes. Significant heterogeneity was evident among the schemes $\left(Q_{(11)}=47.44, P<.0001 ; I^{2}=84.90 \%\right)$; however, the sensitivity analysis did not reveal any influential schemes. Considering the estimate for pre-ERS MET minutes per week (676 MET min), it would seem that the estimate for the change in MET minutes per week resulted in the participants beginning as moderately active and, though their activity levels increased and categorical analysis showed changes, the effect estimate for the change in activity levels was insufficient to result in a change in the IPAQ category, with them remaining moderately active.

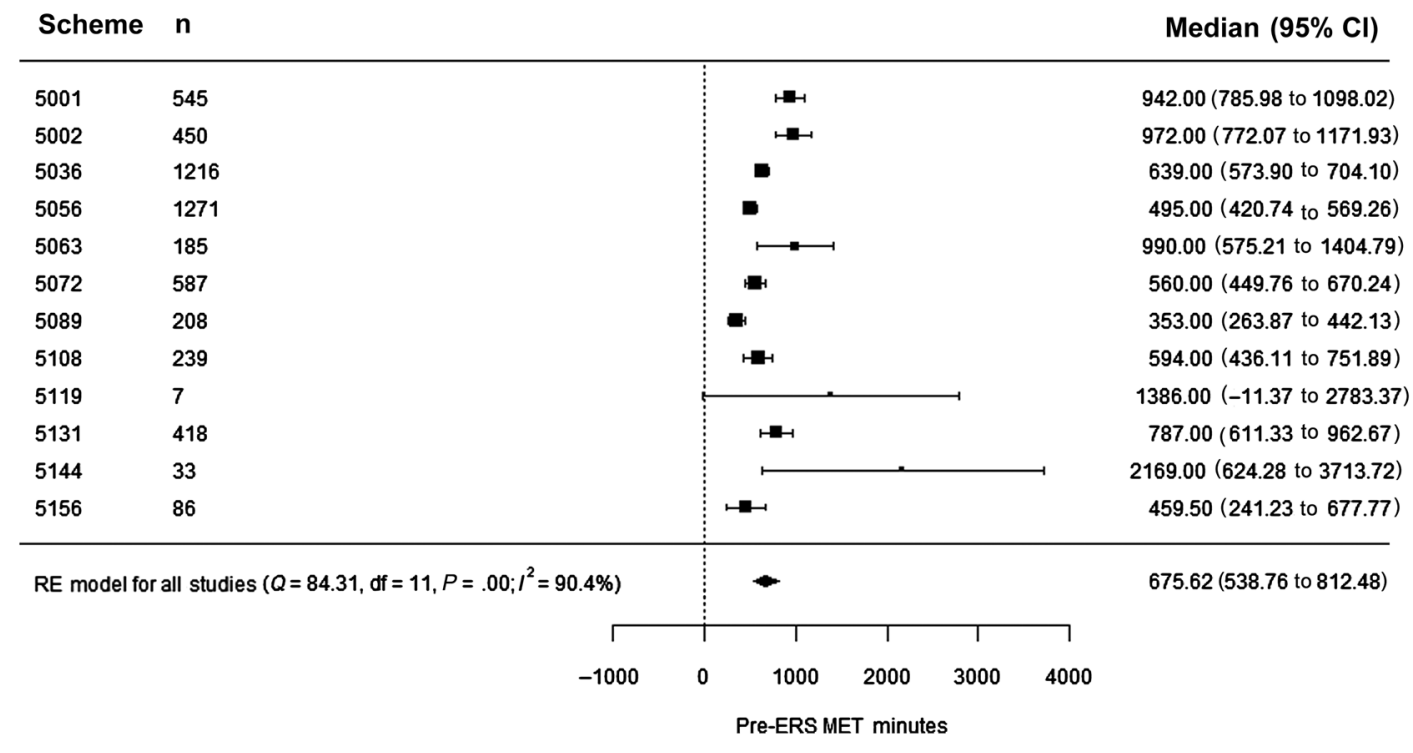

Figure 2 - Forest plot of pre-ERS MET minutes per week across schemes. CI indicates confidence interval; ERS, exercise referral schemes; MET, metabolic equivalent; RE, random effects. 


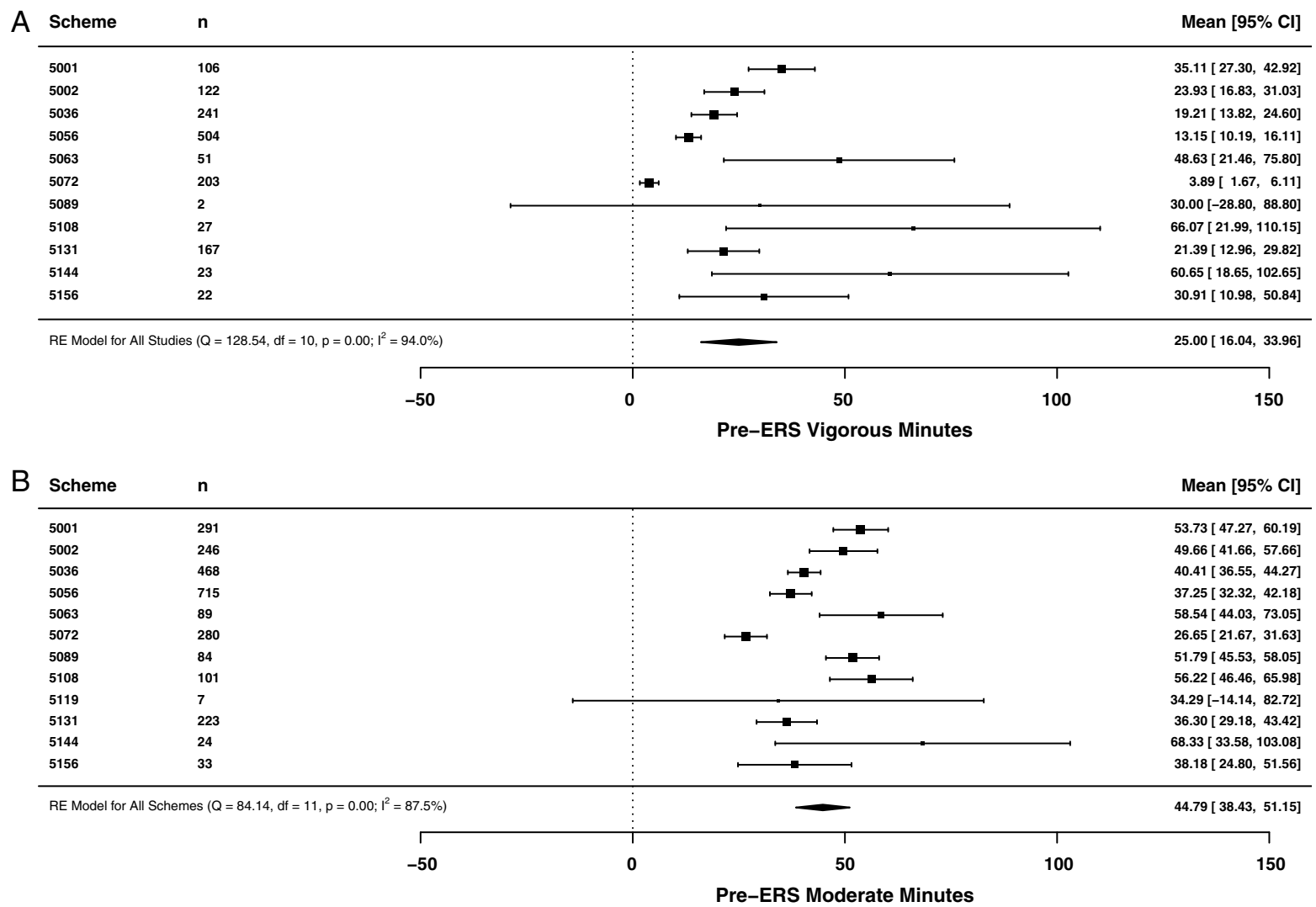

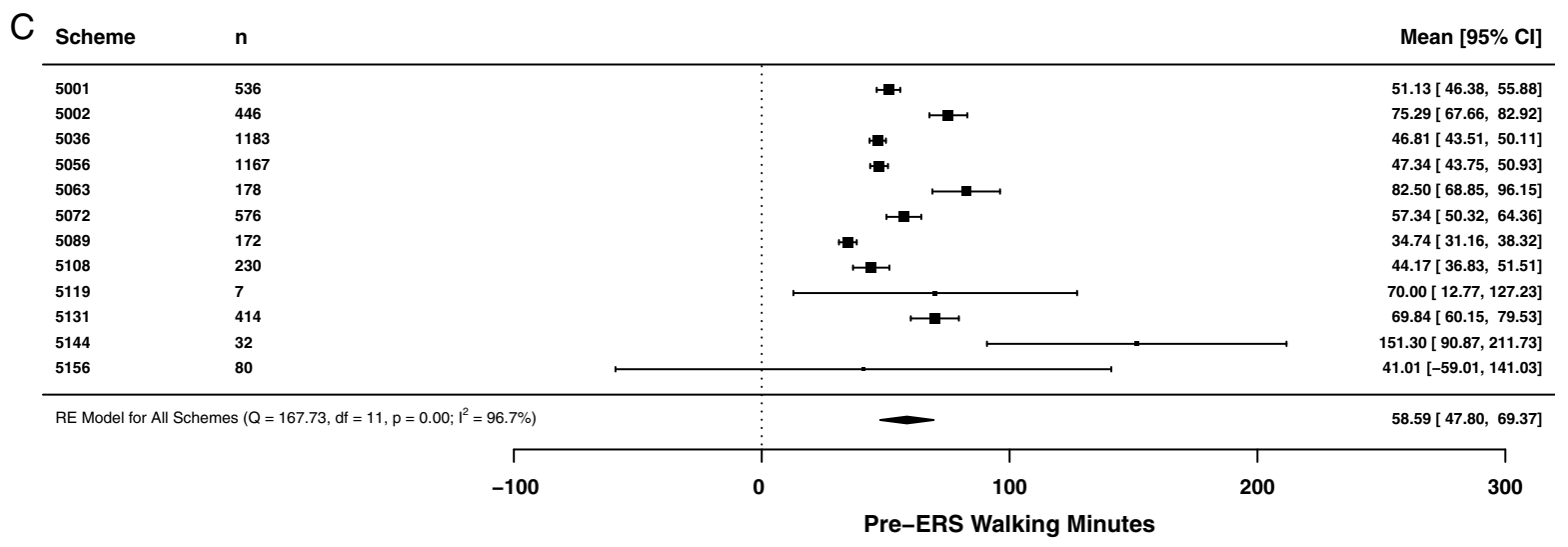

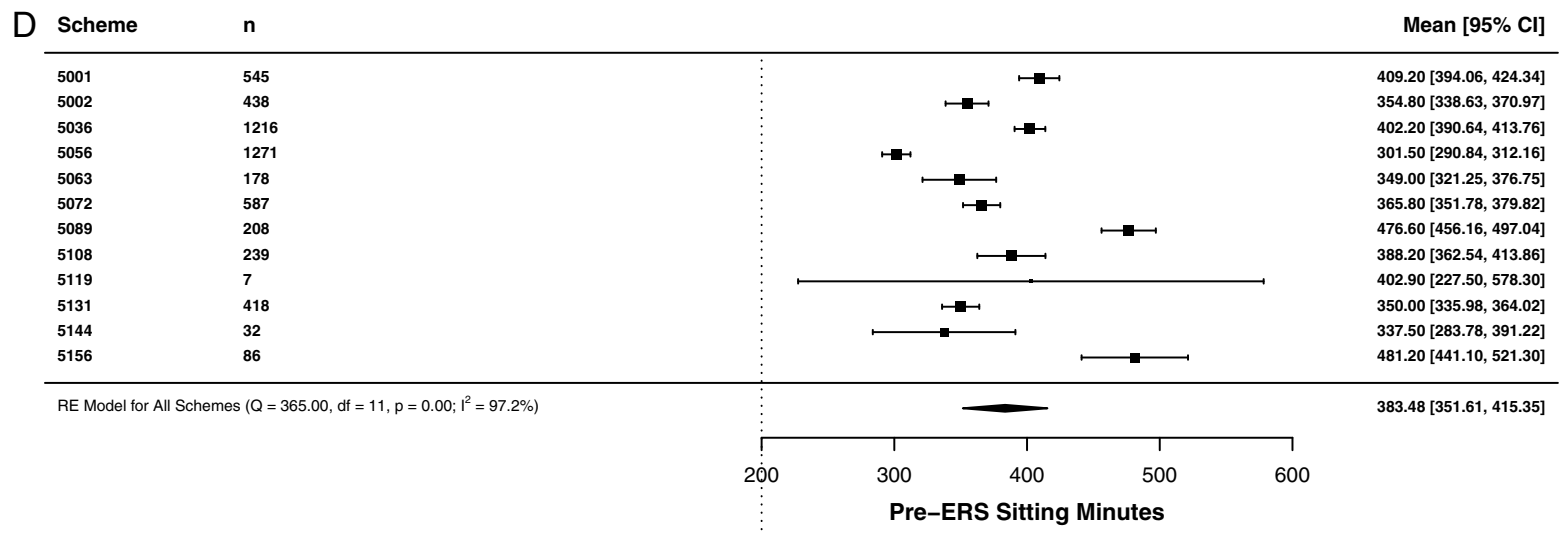

Figure 3 - Forest plot of pre-ERS (A) vigorous, (B) moderate, (C) walking, and (D) sitting minutes across schemes. RE indicates random effects. 


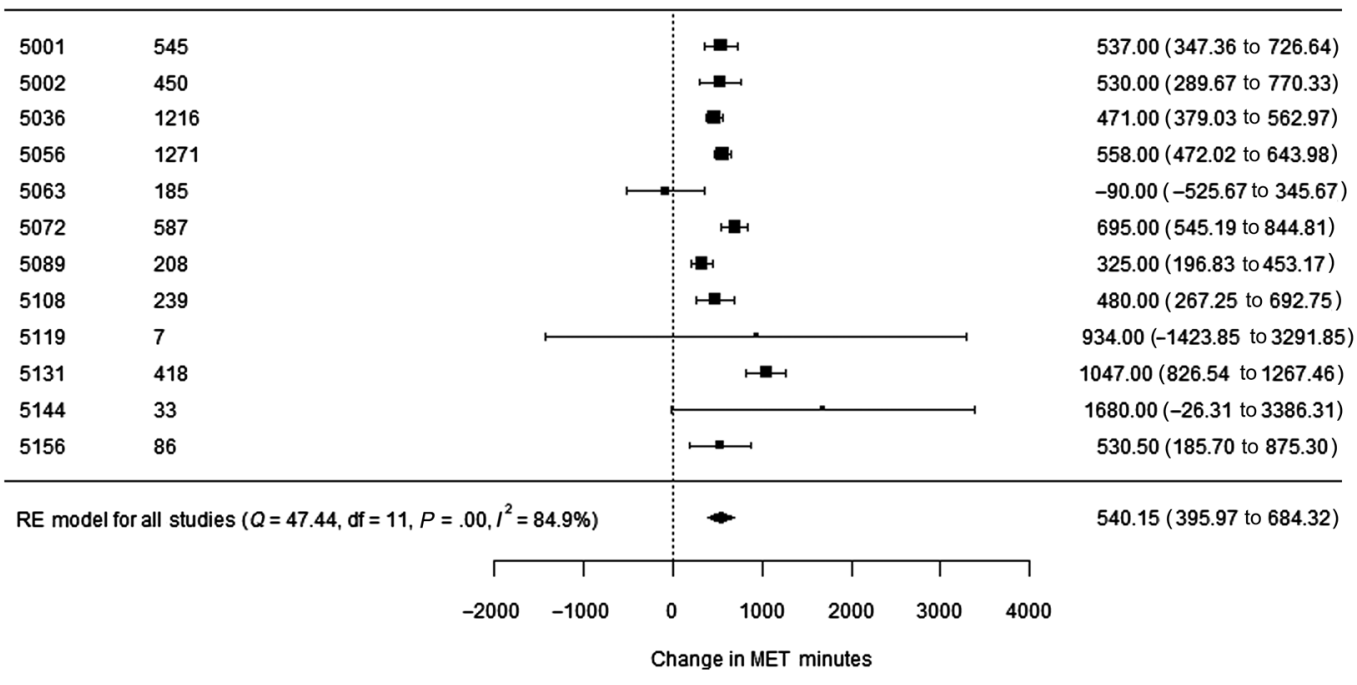

Figure 4 - Forest plot of change in MET minutes per week across schemes. CI indicates confidence interval; MET, metabolic equivalent; RE, random effects.

\section{Breakdown of Change Activity Minutes}

The forest plots are shown for the pre-ERS breakdown of activity minutes in Figure 5. For the change in vigorous activity, the estimate from the random effects model was 17 minutes (9-24 $\min , P<.0001)$. Significant heterogeneity was evident among the schemes $\left(Q_{(11)}=480.16, P<.0001 ; I^{2}=97.87 \%\right)$; however, the sensitivity analysis did not reveal any influential schemes. For the change in moderate activity, the estimate from the random effects model was 29 minutes (22-36 min, $P<.0001)$. Significant heterogeneity was evident among the schemes $\left(Q_{(11)}=133.55, P<\right.$ $.0001 ; I^{2}=92.14 \%$ ); however, the sensitivity analysis did not reveal any influential schemes. For the change in walking, the estimate from the random effects model was -5 minutes $(-14$ to $5 \mathrm{~min}, P=.3687)$. Significant heterogeneity was evident among the schemes $\left(Q_{(11)}=94.79, P<.0001 ; I^{2}=95.91 \%\right)$; however, the sensitivity analysis did not reveal any influential schemes. For the change in sitting, the estimate from the random effects model was -61 minutes $(-78$ to $-43 \mathrm{~min}, P<.0001)$. Significant heterogeneity was evident among the schemes $\left(Q_{(11)}=88.51, P<.0001\right.$; $\left.I^{2}=90.63 \%\right)$; however, the sensitivity analysis did not reveal any influential schemes.

\section{Exploratory Meta-Regression}

Due to the finding that the ERS schemes appear to be attracting those who are categorized as having either "moderate" or "high" PA levels despite guidance suggesting they should be aimed at those who have "low" PA levels, exploratory analysis was performed using mixed effects meta-regression to examine the relationship between the proportion of participants (\%) in each scheme categorized as having "low" PA levels and the changes in the PA levels. The coefficient for change in total MET minutes was not significant $(-5.7 \mathrm{MET} \min [-17.3$ to 5.9$], P=.3347)$, with a significant residual heterogeneity $\left(Q E_{(10)}=39.91, P<.0001\right)$ and $R^{2}=0.00 \%$. Figure 6 shows the meta-analytic scatterplot for the change in total MET minutes and proportion of participants categorized as "low" PA.

The coefficient for change in total vigorous minutes was not significant $(-0.3 \min [-0.9$ to 0.3$], P=.3204)$, with a significant residual heterogeneity $\left(Q E_{(10)}=443.79, P<.0001\right)$ and $R^{2}=0.00 \%$. The coefficient for change in moderate minutes was not significant (0.2 $\min [-0.3$ to 0.7$], P=.4684)$, with a significant residual heterogeneity $\left(Q E_{(10)}=132.00, P<.0001\right)$ and $R^{2}=0.00 \%$. The coefficient for change in walking minutes was not significant $(0.3$ $\min [-0.4$ to 1.1$], P=.3788)$, with a significant residual heterogeneity $\left(Q E_{(10)}=93.03, P<.0001\right)$ and $R^{2}=0.00 \%$. The coefficient for change in sitting minutes was not significant $(0.4 \mathrm{~min}$ [ -0.8 to 1.7$]$, $P=.5230)$, with a significant residual heterogeneity $\left(Q E_{(10)}=75.60\right.$, $P<.0001)$ and $R^{2}=0.00 \%$. Figure 7 shows the meta-analytic scatterplots for change in the breakdown of activity minutes and proportion of participants categorized as "low" PA.

\section{Discussion}

The aim of the present study was to examine the changes in PA in participants who had completed an ERS. This study utilized data from the UK's first National Referral Database. ${ }^{22}$ The categorical data revealed that roughly half of the participants entering the ERSs were classified as having "moderate" or "high" levels of PA. The effect estimates from the meta-analysis showed that the pre-ERS participants" total PA fell in the "moderate" category, completing a median 676 MET minutes per week (539-812 min), composed of 25 minutes (16-34 min) vigorous activity, 45 minutes (38-51 min) moderate activity, 59 minutes (48-69 min) walking, and $384 \mathrm{~min}$ utes (352-415 min) sitting. Significant increases of 540 MET minutes per week (396-684 min) occurred in participants undergoing ERSs, and this change occurred as a result of increases in vigorous activity of 17 minutes (9-24 min), increases in moderate activity of 29 minutes (22-36 min), and reductions in sitting time of -61 minutes ( -78 to $-43 \mathrm{~min})$. Little change was reported in the weekly walking minutes ( $-5 \mathrm{~min}$ [-14 to 5$]$ ). Overall changes were primarily facilitated by increased moderate to vigorous PA (MVPA) and reduced sitting. However, though the categorical data showed at the group level positive changes in the proportion of participants within each IPAQ category (ie, fewer in the "low" and more in the "moderate" and "high" categories), visualization of individual data from pre-ERS to post-ERS showed that changes occurred in both directions with some participants moving to 
RE Model for All Schemes $\left(Q=480.16, d f=11, p=0.00 ; l^{2}=97.9 \%\right)$

$24.14[20.80,27.48]$ 11.35 [ $7.66,15.04]$ 5.81 [ $4.08,7.55]$ $26.52[24.34,28.70]$ $-0.78[-8.96,7.40]$ $16.44[13.21,19.67]$

$4.11[1.55,6.67]$ $8.92[2.65,15.19]$ $8.92[2.65,15.19]$
$47.14[-0.19,94.47]$ $41.56[37.20,45.92]$ $28.79[-3.35,60.93]$ 18.60 [ $2.16,35.04]$

$16.84[9.16,24.52]$

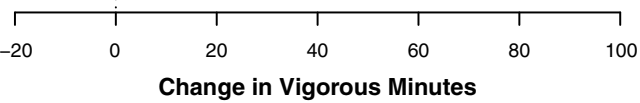

B

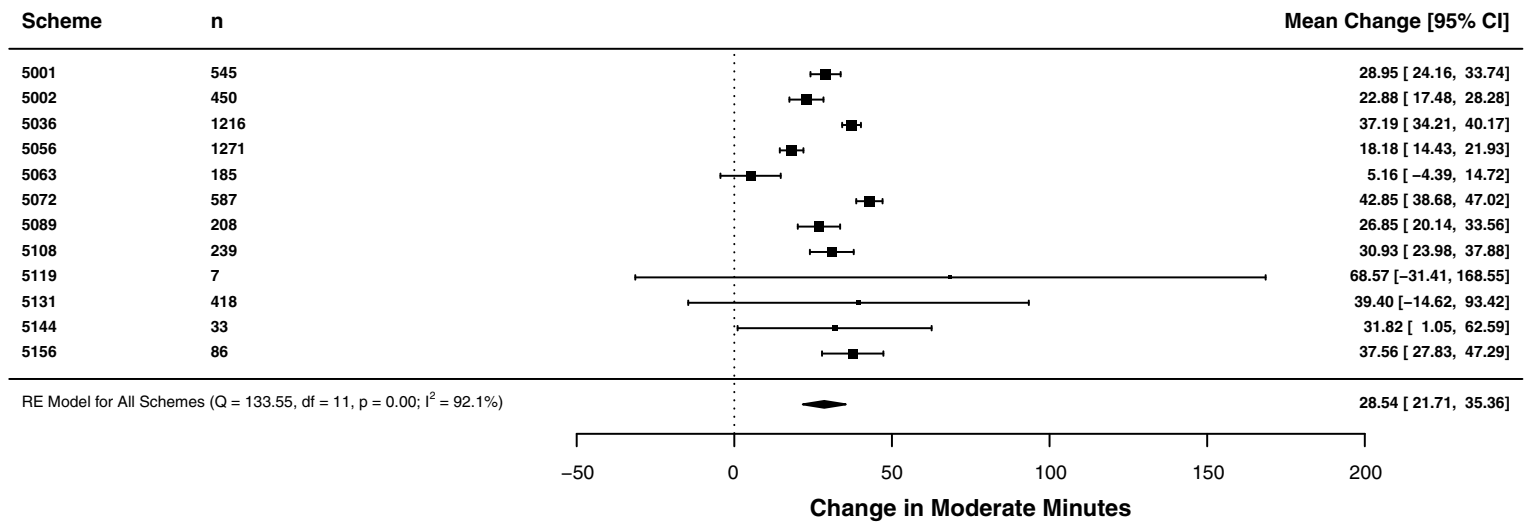

C scheme n

Mean Change $[95 \% \mathrm{Cl}]$

\begin{tabular}{ll}
\hline 5001 & 5 \\
5002 & 450 \\
5036 & 121 \\
5056 & 1 \\
5063 & 18 \\
5072 & 587 \\
5089 & 2 \\
5108 & 239 \\
5119 & 7 \\
5131 & 418 \\
5144 & 33 \\
5156 & 8 \\
\hline
\end{tabular}

545
450
1216
1271
185
587
208
239
7
418
33
86

9.14 [ $3.57,14.70]$ $6.38[-1.97,14.74]$ $4.00[0.43,7.56]$ $4.00[0.43,7.56]$
$-7.41[-11.18,-3.64]$ $-52.25[-66.14,-38.36]$ $7.74[-0.72,16.20]$ 0.31 [ $-3.56,4.19]$ $-3.10[-9.80,3.59]$ $-16.43[-72.39,39.53]$ $-16.43[-72.39,39.53]$
$-5.04[-13.77,3.68]$ $-31.97[-73.52,9.58]$ 2.95 [ $-6.88,12.79]$

RE Model for All Schemes $\left(Q=94.79, d f=11, p=0.00 ; I^{2}=95.9 \%\right)$

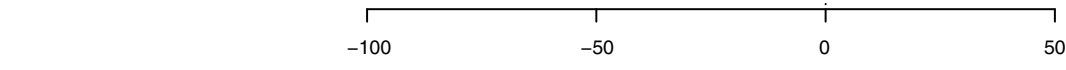

$-4.45[-14.16,5.26]$

Change in Walking Minutes

D

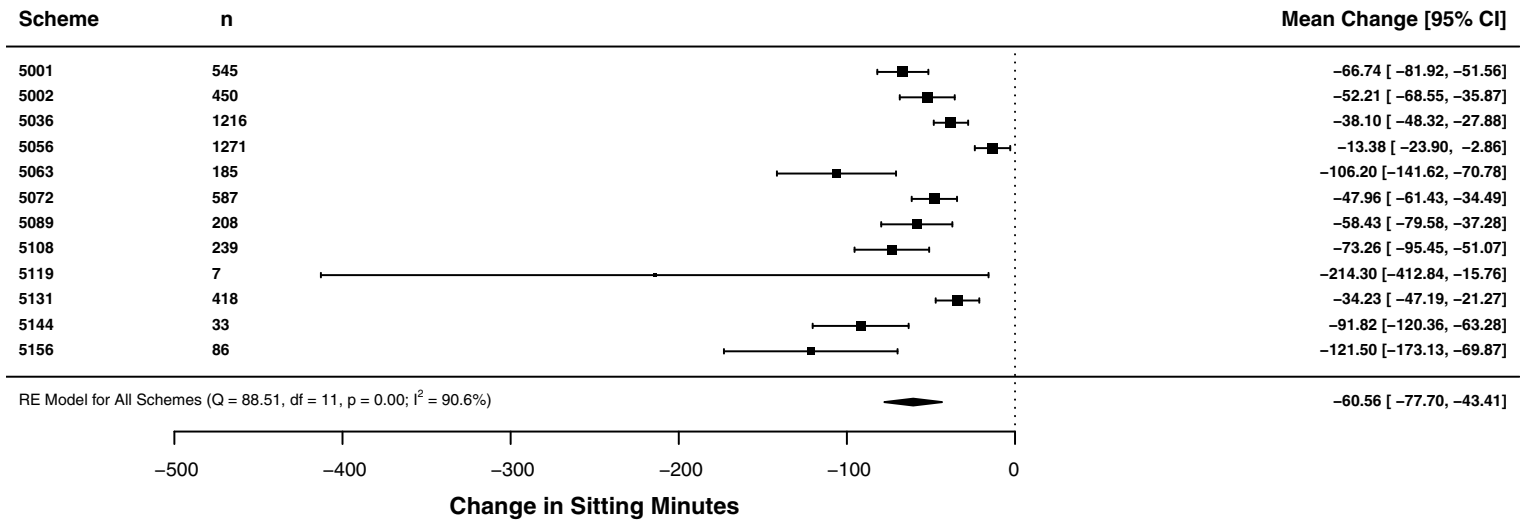

Figure 5 - Forest plot of change in (A) vigorous, (B) moderate, (C) walking, and (D) sitting minutes across schemes. RE indicates random effects. 


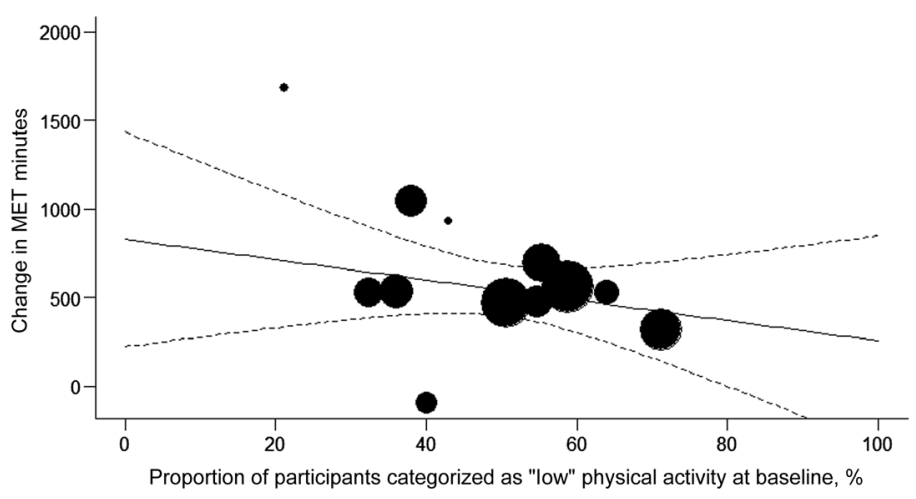

Figure 6 - Meta-analytic scatterplot for change in total MET minutes and proportion of participants (in percentage) categorized as "low" physical activity. MET indicates metabolic equivalent.

higher and lower categories. Consideration of the meta-analytic effect estimate for change would suggest that this was not sufficient to result in categorical change within the population, and participants were, on average, still classed as "moderately active."

Research suggests that a dose-response relationship occurs between PA and health benefits if individuals can improve MET minutes per week by 500 to $1000 \mathrm{MET} \mathrm{min} / \mathrm{week}^{26} \mathrm{Here}$, the participants' change, though statistically significant, barely achieved this threshold, which may explain the small changes observed in the health and well-being outcomes in persons undergoing ERSs. ${ }^{21}$ It is also thought that the dose-response curve for PA is steepest at the lowest end of the curve,${ }^{27}$ that is, moving from a "no" or "low" to a "moderate" PA level. However, the metaanalytic estimate suggested that participants in this study tended to be already moderately active at the beginning of their ERSs. Indeed, in previous studies, some proportion of participants undergoing ERSs have reported themselves as being "moderately inactive" $\left(15.3 \%{ }^{19}\right)$. Chalder et al ${ }^{18}$ also reported that $~ 25 \%$ to $28 \%$ of their participants were already achieving at least 1000 MET minutes per week of PA at the baseline. This is perhaps a cause for concern, as the NICE guidelines ${ }^{13,16}$ suggest that ERSs be targeted toward inactive individuals. The observational data presented here would suggest that this recommendation is not being followed. However, it should be considered that some participants may be overestimating their levels of PA and not accurately recording their activity.

A number of participants moved from the "low" category to both the "moderate" and "high" categories and, considering the dose-response nature of PA changes, it may be that the more meaningful PA, health, and wellness changes primarily occur in those who begin an ERS categorized as inactive. However, exploratory meta-regression analysis examining whether there was an association between the proportion of participants in schemes classified as having "low" PA levels did not demonstrate this. Further, the supplementary analysis performed by Wade et $\mathrm{al}^{21}$ did not show particularly strong relationships between changes in the PA levels and the health and well-being outcomes in ERS participants from the analysis conducted using The National Referral database.

The ERSs can and do increase PA levels; however, the value of this to a participant's health outcomes is less clear. In their systematic review and meta-analysis, Pavey et $\mathrm{al}^{1}$ reported that, compared with usual care, ERSs have a slightly greater impact on the number of participants achieving between 90 and 150 minutes
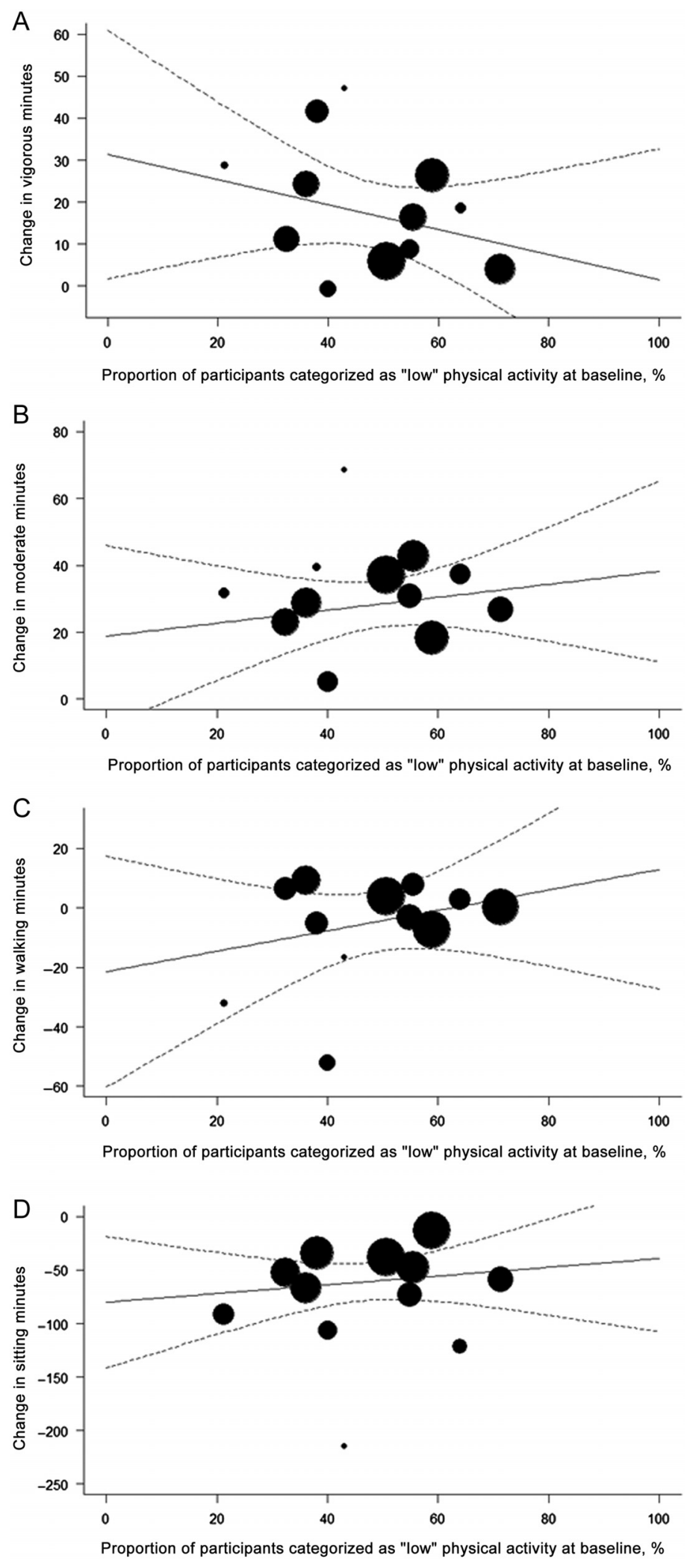

Figure 7 - Meta-analytic scatterplots for change in (A) vigorous, (B) moderate, (C) walking, and (D) sitting minutes and proportion of participants categorized as "low" physical activity. 
of moderate activity per week. However, they noted that, at the time, the evidence was weak. Using a 7-day PA recall, Murphy et $\mathrm{al}^{19}$ found that ERS group participants at 12 months post intervention achieved a median of 200 minutes of exercise compared with 165 for the control group. Chalder et $\mathrm{al}^{18}$ found increases in PA postintervention, though no significant differences, in the proportion of participants meeting at least 1000 MET minutes per week between ERS or usual care in depressed adults, though descriptively, they noted slight differences (ERS $=52 \%$ at $4 \mathrm{mo}, 63 \%$ at $8 \mathrm{mo}$, and $58 \%$ at $12 \mathrm{mo}$; usual care $=43 \%$ at $4 \mathrm{mo}$, $49 \%$ at $8 \mathrm{mo}$, and $40 \%$ at $12 \mathrm{mo}$ ). Our results show that changes likely do occur, although not of considerable magnitude.

It is also worth considering the nature of the change in PA levels. The increases in total PA were primarily driven by increases in MVPA and decreased sitting. The participants increased MVPA per week by $\sim 46$ minutes ( 17 min vigorous and 29 min moderate), yet walking did not change much in the participants undergoing ERSs. Therefore, it could be recommended that schemes should encourage walking outside of an ERS attendance, as there was no change reported in walking post completion. Though walking and light activities are associated with improvements in all-cause mortality, these seem to be greatest again at the lower end of the dose-response curve, ${ }^{28}$ and at an equal volume, MVPA is associated with greater benefits. ${ }^{29}$ It could therefore be viewed as positive that MVPA increased in patients undergoing ERSs. Increases in MVPA, even in small amounts, have been shown to be associated with reductions in all-cause mortality. ${ }^{30}$ Jefferis et al $^{30}$ reported each 10-minute increase in MVPA per day resulted in a $10 \%$ reduction in all-cause mortality risk. O'Donovan et $\mathrm{al}^{31}$ have recently reported that inclusion of vigorous activity has an even stronger impact upon cardiovascular disease mortality risk, and the participants in this present study showed increases in vigorous activity, which may still yield significant health benefits beyond the scope of our timeframe. Furthermore, there was $\sim 1$-hour reduction in sitting time per week across participants. However, recent data show that reducing sitting time-point estimates of $\sim 30$ minutes per day are considered clinically meaningful. ${ }^{32}$

There are several limitations with the current database ${ }^{22}$ that are worthy of note, and these partially extend to the data analysis here. Length of scheme is a factor that could influence changes in PA. However, in this study, the majority of schemes were of 12 weeks (or $3 \mathrm{mo}$ ) in length with one scheme (5056) being 6 weeks long. In a recent systematic review, ${ }^{33}$ it was reported that longer-length schemes (>20 wk) improved adherence to PA prescribed over the course of the scheme. This research emphasizes the importance of increasing the length of schemes. Indeed, it may be that if longer schemes were present in the database for analysis, these may effect greater PA increases compared with shorter schemes. However, other research by Webb et $\mathrm{al}^{20}$ suggests that shorter schemes can be effective, as it was found that, after completing an 8-week ERS, categorical IPAQ scores significantly increased.

Another limitation, considering PA levels specifically, the use of self-reported outcomes is a potential issue. IPAQ is, of course, a subjective measure and was not designed for an examination of change in PA levels, and this could mean it does not well reflect participants' objective changes in $\mathrm{PA}^{24,34}$; however, this was the only measure used within the database reviewing the PA levels of participants. Although, a recent study has suggested that participants' perceptions of their PA levels relative to others, even when independent of the actual PA conducted (whether self-reported or device measured), are strong predictors of all-cause mortality. ${ }^{35}$ Although this study reviewed the effects of ERSs on change in PA, it does not consider the reasons why the participants chose to attend an ERS. Indeed, many factors influence uptake, ${ }^{36}$ and it seems likely they would influence engagement throughout also. Some participants may have attended due to their own motivation to improve their health conditions, whereas other participants may have only attended because their general practitioner advised them to. A future study could review the reasoning behind individuals' uptake in schemes, along with recorded adherence to PA or self-report PA through IPAQ. This could also be captured by schemes within the database as it is developed. Lastly, similar to the health and well-being outcomes, there was considerable heterogeneity across the schemes with respect to the changes observed.

\section{Conclusions}

These results represent the initial findings from the first analysis of the National Referral Database considering PA levels. The analyses performed here were with the intention of considering broadly "do we observe a change in PA in individuals who are undergoing ERSs?" and the findings suggest that significant changes in total MET minutes per week do occur. The participants in the ERSs assessed here were, however, predominantly "moderately active" at the baseline and remained so post-ERS. Thus, it is not clear the degree to which the changes observed are meaningful or not. Considering the heterogeneity of the results across schemes also, future work, including that afforded by this database, should be focused upon determining where best practice exists (ie, what works best for which population).

\section{Acknowledgments}

The authors would like to thank Alan Batterham for his insight regarding the statistical approaches taken to analyzing the data presented here. There was no patient or public involvement in the production of this research. All data is available upon request from the corresponding author, and we would encourage researchers to consider broader questions that might be answered with this data set and to contact us in this regard. As per the Health Research Authority and research ethics committee section 11 of Standard Operating Procedures, ethical approval is not required for research involving patient data that is not identifiable. However, as this work was conducted as part of a $\mathrm{PhD}$ project, local ethics approval was obtained from Coventry University (P46119). N.R. received joint funding for a $\mathrm{PhD}$ studentship from Coventry University and ukactive. The authors declare no conflicts of interest.

\section{References}

1. Pavey TG, Fox K, Hillsdon M, et al. Effect of exercise referral schemes in primary care on physical activity and improving health outcomes: systematic review and meta-analysis. BMJ. 2011;343: d6462. PubMed ID: 22058134 doi:10.1136/bmj.d6462

2. Pedersen BK, Saltin B. Evidence for prescribing exercise as therapy in chronic disease. Scand J Med Sci Sports. 2006;16(suppl 1):3-63. doi:10.1111/j.1600-0838.2006.00520.x

3. Pedersen BK, Saltin B. Exercise as medicine-evidence for prescribing exercise as therapy in 26 different chronic diseases. Scand J Med Sci Sports. 2015;25(suppl 3):1-72. doi:10.1111/sms.12581

4. World Health Organization. Information Sheet: Global Recommendations on Physical Activity for Health 18-64 Years Old. Geneva, Switzerland: World Health Organization; 2011. https://www.who.int/ 
dietphysicalactivity/publications/recommendations18_64yearsold/ en/. Accessed January 3, 2019.

5. Booth FW, Roberts CK, Laye MJ. Lack of exercise is a major cause of chronic diseases. Compr Physiol. 2012;2(2):1143-1211. PubMed ID: 23798298

6. British Heart Foundation. Physical Inactivity and Sedentary Behaviour Report 2017. London, UK: British Heart Foundation; 2017. https://www.bhf.org.uk/informationsupport/publications/statistics/ physical-inactivity-report-2017. Accessed January 3, 2019.

7. Sport England. Active Lives. London, UK: Sport England; 2018. https://www.sportengland.org/research/active-lives-survey/. Accessed January 3, 2019.

8. Morgan F, Battersby A, Weightman AL, et al. Adherence to exercise referral schemes by participants-what do providers and commissioners need to know? A systematic review of barriers and facilitators. BMC Public Health. 2016;16(1):227. doi:10.1186/s12889-0162882-7

9. Public Health England. Physical Activity. London, UK: Public Health England; 2016. http://fingertips.phe.org.uk/profile/physicalactivity/data\#page/0/gid/1938133004/pat/6/par/E12000005/ati/102/ are/E08000029/iid/91494/age/44/sex/4. Accessed January 3, 2019.

10. Naci H, Ioannidis JPA. Comparative effectiveness of exercise and drug interventions on mortality outcomes: metaepidemiological study. BMJ. 2013;347:f557. doi:10.1136/bmj.f5577

11. Naci H, Salcher-Konrad M, Dias S, et al. How does exercise treatment compare with antihypertensive medications? A network meta-analysis of 391 randomised controlled trials assessing exercise and medication effects on systolic blood pressure. Br J Sports Med. 2019; 53(14):859-869. doi:10.1136/bjsports-2018-099921

12. Hanson CL, Allin LJ, Ellis JG, Dodd-Reynolds CJ. An evaluation of the efficacy of the exercise on referral scheme in Northumberland, UK: association with physical activity and predictors of engagement. A naturalistic observation study. BMJ Open. 2013;3(8):e002849. PubMed ID: 23913772 doi:10.1136/bmjopen-2013-002849

13. National Institute for Health and Care Excellence (NICE). Physical Activity: Exercise Referral Schemes (PH54). London, UK: NICE; 2014. https://www.nice.org.uk/guidance/ph54

14. Williams N, Hendry M, France B. Effectiveness of exercise referral schemes to promote physical activity in adults: a systematic review. Br J Gen Pract. 2007;57:979-986. PubMed ID: 18252074 doi:10. 3399/096016407782604866

15. Fox K, Biddle S, Edmunds L. Physical activity promotion through primary health care in England. Br J Gen Pract. 1997;47:367-369. PubMed ID: 9231471

16. National Institute for Health and Care Excellence (NICE). Physical Activity: Exercise Referral Schemes (PH54). London, UK: NICE; 2018. https://www.nice.org.uk/guidance/ph54/resources/physicalactivity-exercise-referral-schemes-pdf-1996418406085. Accessed January 3, 2019.

17. Henderson HE, Evans AB, Allen-Collinson J, Siriwardena NA. The 'wild and wooly' world of exercise referral schemes: contested interpretations of an exercise as medicine programme. Qual Res Sport Exerc Health. 2018;10:4.

18. Chalder M, Wiles NJ, Campbell J, et al. Facilitated physical activity as a treatment for depressed adults: Randomised controlled trial. BMJ. 2012;344:e2758. PubMed ID: 22674921 doi:10.1136/bmj.e2758

19. Murphy S, Tudor-Edwards R, Williams N, et al. An evaluation of the effectiveness and cost effectiveness of the National Exercise Referral Scheme in Wales, UK: a randomised controlled trial of a public health policy initiative. J Epidemiol Community Health. 2012;66:745-753.

20. Webb R, Thompson JES, Ruffino JS, et al. Evaluation of cardiovascular risk-lowering health benefits accruing from laboratory-based, community-based and exercise-referral exercise programmes. BMJ Open Sport Exerc Med. 2016;2(1):e000089. PubMed ID: 27900165 doi:10.1136/bmjsem-2015-000089

21. Wade M, Mann S, Copeland R, Steele J. Effect of exercise referral schemes upon health and wellbeing: initial observational insights using individual patient data meta-analysis from the National Referral Database. J Epidemiol Community Health. 2020;74(1):32-41. PubMed ID: 31740446 doi:10.1136/jech-2019-212674

22. Steele J, Wade M, Polley M, Copeland R, Stokes S, Mann S. The National Referral Database: an initial overview. SportRxiv. 2019. doi:10.31236/osf.io/rgywq

23. Craig CL, Marshall AL, Sjöström M, et al. International physical activity questionnaire: 12-country reliability and validity. Med Sci Sports Exerc. 2003;35(8):1381-1395. PubMed ID: 12900694 doi:10. 1249/01.MSS.0000078924.61453.FB

24. Lee PH, Macfarlane DJ, Lam TH, Stewart SM. Validity of the international physical activity questionnaire short form (IPAQ-SF): a systematic review. Int J Behav Nutr Phys Act. 2011;8:115. PubMed ID: 22018588 doi:10.1186/1479-5868-8-115

25. Ainsworth B, Haskell WL, Hermann SD, et al. 2011 compendium of physical activities: a second update of codes and MET values. Med Sci Sports Exerc. 2011;43(8):1575-1581. PubMed ID: 21681120 doi:10.1249/MSS.0b013e31821ece12

26. Nelson ME, Rejeski WJ, Blair SN, et al. Physical activity and public health in older adults: recommendation from the American College of Sports Medicine and the American Heart Association. Med Sci Sports Exerc. 2007;39(8):1435-1445. PubMed ID: 17762378 doi:10.1249/ mss.0b013e3180616aa2

27. Wasfy M, Baggish AL. Exercise dose in clinical practice. Circulation. 2016;133(23):2297-2313. PubMed ID: 27267537 doi:10.1161/ CIRCULATIONAHA.116.018093

28. Kelly P, Kahlmeier S, Götschi T, et al. Systematic review and metaanalysis of reduction in all-cause mortality from walking and cycling and shape of dose response relationship. Int J Behav Nutr Phys Act. 2014;11:132. PubMed ID: 25344355 doi:10.1186/s12966-0140132-x

29. Saint-Maurice PF, Troiana RP, Berrigan D, Kraus WE, Matthews CE. Volume of light versus moderate-to-vigorous physical activity: similar benefits for all-cause mortality? J Am Heart Assoc. 2018;7(7): e008815. PubMed ID: 29610219

30. Jefferis BJ, Parsons TJ, Sartini C, et al. Objectively measured physical activity, sedentary behaviour and all-cause mortality in older men: does volume of activity matter more than pattern of accumulation? $\mathrm{Br}$ J Sports Med. 2019;53(16):1013-1020. doi:10.1136/bjsports-2017098733

31. O’Donovan G, Stamatakis E, Stensel DJ, Hamer M. The importance of vigorous-intensity leisure-time physical activity in reducing cardiovascular disease mortality risk in the obese. Mayo Clin Proc. 2018;93(8):1096-1103.

32. Peachey MM, Richardson J, Tang A, Dal-Bello Haas V, Gravesande J. Environmental, behavioural and multicomponent interventions to reduce adults' sitting time: a systematic review and meta-analysis. Br J Sports Med. 2020;54(6):315-325. doi: 10.1136/bjsports-2017098968

33. Rowley N, Mann S, Steele J, Horton E, Jimenez A. The effects of exercise referral schemes in the United Kingdom in those with cardiovascular, mental health, and musculoskeletal disorders: an updated systematic review. BMC Public Health. 2018;18(1):949. PubMed ID: 30068338 doi:10.1186/s12889-018-5868-9

34. Limb ES, Ahmad S, Cook DG, et al. Measuring change in trials of physical activity intervention: a comparison of self-report questionnaire and accelerometry within the PACE-UP trial. Int J Behav Nutr 
Phys Act. 2019;16:10. PubMed ID: 30670036 doi:10.1186/s12966018-0762-5

35. Zahrt OH, Crum AJ. Perceived physical activity and mortality: evidence from three nationally representative US samples. Health Psychol. 2017;26(11):1017-1025. doi:10.1037/hea0000531
36. Birtwistle SB, Ashcroft G, Murphy R, Gee I, Poole H, Watson PM. Factors influencing patient uptake of an exercise referral scheme: a qualitative study. Health Educ Res. 2019;34(1):113-127. PubMed ID: 30307496 doi:10.1093/her/cyy038 